\title{
Exponential estimates for the Wiener sausage
}

\author{
M. van den Berg and B. Tóth * \\ Department of Mathematics, Heriot-Watt University, Edinburgh, Riccarton, EH14 4AS, \\ United Kingdom
}

Received May 15, 1990; in revised form September 25, 1990

Summary. Let $C_{\varepsilon}(t)$ be the Wiener sausage of radius $\varepsilon$ in $\mathbf{R}^{d}$ up to time $t$. We obtain bounds on the asymptotics of $\mathbf{E} \exp \left(\lambda\left|C_{\varepsilon}(t)\right|\right)$ as $t \rightarrow \infty$, for all $\lambda>0$.

\section{Introduction and results}

Let $(w(t), t \geqq 0)$ be a Wiener process in euclidean space $\mathbf{R}^{d}(d=2,3, \ldots)$ with $w(0)=0$ and generator $\Delta$, the Laplacian for $\mathbf{R}^{d}$. The Wiener sausage of radius $\varepsilon>0$ associated to this process up to time $t$, is the set

$$
C_{\varepsilon}(t)=\left\{y \in \mathbf{R}^{d}\left|\inf _{0 \leqq s \leqq t}\right| y-w(s) \mid<\varepsilon\right\} .
$$

$C_{\varepsilon}(t)$ is Lebesgue measurable with probability 1 . We denote its measure by $\left|C_{\varepsilon}(t)\right|$.

The asymptotics of $\mathbf{E} \exp \left(-\lambda\left|C_{\varepsilon}(t)\right|\right)$ as $t \rightarrow \infty$ for $\lambda>0$ has been of interest to many authors (see e.g. [5], [10], [11] and references cited therein). In [5] it has been shown that for all $\lambda>0$

(1.2) $\lim _{t \rightarrow \infty} t^{-\frac{d}{d+2}} \log \mathbf{E} \exp \left(-\lambda\left|C_{\varepsilon}(t)\right|\right)=-\frac{d+2}{d} \omega_{d}^{2 /(d+2)}\left(\begin{array}{c}2 \lambda_{1} \\ d\end{array}\right)^{d /(d+2)} \lambda^{2 /(d+2)}$,

where $\omega_{d}$ is the volume of the ball of radius 1 in $\mathbf{R}^{d}$

$$
\omega_{d}=\frac{\pi^{d / 2}}{\Gamma\left(1+\frac{d}{2}\right)},
$$

and $-\lambda_{1}$ is the highest eigenvalue of the Dirichlet Laplacian on a ball of radius 1 in $\mathbf{R}^{d}$.

* On leave from the Mathematical Institute of the Hungarian Academy of Sciences, Reáltanoda u. 13-15, H-1053 Budapest 
In this paper we obtain results for the large time asymptotics of $\mathbf{E} \exp ($ $\left.+\lambda\left|C_{\varepsilon}(t)\right|\right)$ for $\lambda>0$. It follows from a standard subadditivity argument that the limit

$$
\lim _{t \rightarrow \infty} \frac{1}{t} \log \mathbf{E} \exp \left(\lambda\left|C_{\varepsilon}(t)\right|\right)=\inf _{t>0} \frac{1}{t} \log \mathbf{E} \exp \left(\lambda\left|C_{\varepsilon}(t)\right|\right)=S(\lambda, \varepsilon) \in[0, \infty]
$$

exists for any $\lambda$. (However, we know from [5] that this limits is identically zero for $\lambda<0$.) Furthermore, by scaling one can see that

$$
S(\lambda, \varepsilon)=\varepsilon^{-2} S\left(\varepsilon^{d} \lambda, 1\right),
$$

so that we shall study only the case $\varepsilon=1$. Define

$$
S(\lambda, 1)=S(\hat{\lambda}) .
$$

As a lim inf of convex continuous functions, $\lambda \mapsto S(\lambda)$ itself is convex and lower semicontinuous.

To our knowledge this problem has been considered in two papers only. It has been shown that $S(\lambda)=\lambda^{2}$ in the one-dimensional case [4]. Furthermore, it has been shown that $\mathbf{E} \exp \left(+\lambda\left|C_{\varepsilon}(t)\right|\right)$ is finite for all $\lambda>0$ and $t>0$ [9]. In this paper we complement the results of [4] and [9] by obtaining upper and lower bounds for $S(\lambda)$ in two and more dimensions. We include for the sake of completeness the one-dimensional case in the main result of this paper:

Theorem 1. The limit (1.4) is positive and finite in any dimension and for any $\lambda>0$. (i) In one dimension

$$
S(\lambda)=\lambda^{2}
$$

(ii) In two dimensions

$$
4 \lambda^{2} \leqq S(\lambda) \leqq 16 e^{4} \max \left\{\lambda, \lambda^{2}\right\} .
$$

(iii) In three and more dimensions

$$
\max \left\{\omega_{d-1}^{2} \lambda^{2}, d(d-2) \omega_{d} \lambda\right\} \leqq S(\lambda) \leqq 2^{d} d \omega_{d-1} \mathrm{e}^{2^{d-1} \omega_{d-1}} \max \left\{\lambda, \lambda^{2}\right\} .
$$

It is not difficult to obtain slightly better constants in the upper bounds of Theorem 1 , in the asymptotic regimes $\lambda \gg 1$ and $\lambda \ll 1$. However, the sharp behaviour can not be caught with our method.

One can see that the sign in the exponent in (1.2) respectively (1.4) makes essential difference. Due to the minus sign the asymptotics in (1.2) is determined by the large deviations of the volume of the Wiener sausage towards small nontypical values. And this is the phenomenological explanation of the fact that the asymptotics in (1.2) is exactly the same as that of $\mathbf{E} \exp \left(-\lambda \omega_{d} m(t)^{d}\right)$ where $m(t)$ is the maximum displacement of the Wiener process. Both asymptotics are determined by nontypical small values of $\left|C_{z}(t)\right|$ and $m(t)$ respectively, and a Wiener process conditioned to a small nontypical $m(t)$ fills completely the sphere of radius $m(t)$. Thus the trivial upper bound

$$
\left|C_{\varepsilon}(t)\right| \leqq \omega_{d}(m(t)+\varepsilon)^{d}
$$


becomes sharp in this non-typical regime.

On the other hand the large time asymptotics of $\mathbf{E} \exp \left(+\lambda\left|C_{\varepsilon}(t)\right|\right)$ is dominated by large deviations towards large non-typical values of $\left|C_{\varepsilon}(t)\right|$. A first phenomenological guess would be that once more the asymptotics of a trivial bound

$$
\left|C_{\varepsilon}(t)\right| \geqq \omega_{d-1} \varepsilon^{d-1} m(t)+\omega_{d} \varepsilon^{d}
$$

becomes sharp, since both asymptotics are dominated by non-typically long Brownian excursions. As one can see from the main result, the situation is more complicated.

The structure of the present note is the following: in Sect. 2 the explicit calculations are presented for the one dimensional case. In Sect. 3 the lower bounds are proved. Finally Sect. 4 is devoted to the upper bounds in Theorem 1.

\section{Proof of Theorem 1 (i)}

Consider the Wiener process $w(t)$ on $\mathbf{R}$, with $w(0)=0$. Let

$$
r(t)=\max _{0 \leqq s \leqq t} w(s)-\min _{0 \leqq s \leqq t} w(s),
$$

be the range of the Wiener process up to time $t$. Then for $d=1$

$$
\mathbf{E} \mathrm{e}^{\lambda\left|C_{1}(t)\right|}=\mathbf{E} \mathrm{e}^{\lambda(r(t)+2)}
$$

The density of the random variable $r(t)$ has been computed in [1] and is given by

$$
f(p, t)= \begin{cases}\frac{4}{(\pi t)^{1 / 2}} \sum_{k=1}^{\infty}(-1)^{k-1} k^{2} \mathrm{e}^{-p^{2} k^{2} /(4 t)}, & p \geqq 0, \\ 0, & p<0 .\end{cases}
$$

To prove Theorem 1(i) we first obtain a lower bound for $\mathbf{E e}^{\lambda\left|C_{1}(t)\right|}$. For $p>2 t^{1 / 2}$ $k^{2} \mathrm{e}^{-p^{2} k^{2} /(4 t)}$ is strictly decreasing in $k$. Hence

$$
f(p, t) \geqq \frac{4}{(\pi t)^{1 / 2}}\left\{\mathrm{e}^{-p^{2} /(4 t)}-4 \mathrm{e}^{-p^{2} / t}\right\} \geqq \frac{4}{(\pi t)^{1 / 2}}\left(1-\frac{4}{e^{3}}\right) \mathrm{e}^{-p^{2} /(4 t)},
$$

for $p \geqq 2 t^{1 / 2}$. For $t \geqq \lambda^{-2}$ we have by (2.4)

$$
\begin{aligned}
\mathrm{Ee}^{\lambda\left|C_{1}(t)\right|} & \geqq \mathrm{e}^{2 \lambda} \int_{2 t^{1 / 2}}^{\infty} \mathrm{d} p \mathrm{e}^{\lambda p} f(p, t) \geqq \mathrm{e}^{2 \lambda} \int_{2 t^{1 / 2}}^{\infty} \mathrm{d} p \mathrm{e}^{\lambda p} \frac{4}{(\pi t)^{1 / 2}}\left(1-\frac{4}{e^{3}}\right) \mathrm{e}^{-p^{2} /(4 t)} \\
& \geqq \mathrm{e}^{2 \lambda} \int_{2 t \lambda}^{\infty} \mathrm{d} p \mathrm{e}^{\lambda p} \frac{4}{(\pi t)^{1 / 2}}\left(1-\frac{4}{e^{3}}\right) \mathrm{e}^{-p^{2} /(4 t)}=\mathrm{e}^{t \lambda^{2}+2 \lambda}\left(2-\frac{8}{e^{3}}\right) \geqq \mathrm{e}^{t \lambda^{2}}
\end{aligned}
$$


This proves that

$$
\liminf _{t \rightarrow \infty} \frac{1}{t} \log \mathbf{E} \mathrm{e}^{\lambda\left|C_{1}(t)\right|} \geqq \lambda^{2}
$$

Next we obtain an upper bound for $\mathbf{E}^{\lambda\left|C_{1}(t)\right|}$.

$$
\begin{aligned}
\mathbf{E e}^{\lambda\left|C_{1}(t)\right|} & =\mathrm{e}^{2 \lambda}\left\{\int_{0}^{2 t^{1 / 2}} \mathrm{~d} p \mathrm{e}^{\lambda p} f(p, t)+\int_{2 t^{1 / 2}}^{\infty} \mathrm{d} p \mathrm{e}^{\lambda p} f(p, t)\right\} \\
& \leqq \mathrm{e}^{2 \lambda+2 t^{1 / 2} \lambda} \int_{0}^{2 t^{1 / 2}} \mathrm{~d} p f(p, t)+\mathrm{e}^{2 \lambda} \int_{2 t^{1 / 2}}^{\infty} \mathrm{d} p \mathrm{e}^{\lambda p} f(p, t) \\
& \leqq \mathrm{e}^{2 \lambda+2 t^{1 / 2} \lambda}+\mathrm{e}^{2 \lambda} \int_{2 t^{1 / 2}}^{\infty} \mathrm{d} p \mathrm{e}^{\lambda p} f(p, t) .
\end{aligned}
$$

Moreover, for $p \geqq 2 t^{1 / 2}$

$$
f(p, t) \leqq \frac{4}{(\pi t)^{1 / 2}} \mathrm{e}^{-p^{2} /(4 t)}
$$

Hence, by (2.7) and (2.8)

$$
\begin{aligned}
\mathrm{Ee}^{\lambda\left|C_{1}(t)\right|} & \leqq \mathrm{e}^{2 \lambda+2 t^{1 / 2} \lambda}+\mathrm{e}^{2 \lambda} \int_{2 t^{1 / 2}}^{\infty} \mathrm{d} p \mathrm{e}^{\lambda p-p^{2} /(4 t)} 4(\pi t)^{-1 / 2} \\
& \leqq \mathrm{e}^{2 \lambda+2 t^{1 / 2} \lambda}+\mathrm{e}^{2 \lambda} \int_{-\infty}^{\infty} \mathrm{d} p \mathrm{e}^{\lambda p-p^{2} /(4 t)} 4(\pi t)^{-1 / 2}=\mathrm{e}^{2 \lambda+2 t^{1 / 2} \lambda}+8 \mathrm{e}^{2 \lambda+\lambda^{2} t}
\end{aligned}
$$

This proves that

$$
\limsup _{t \rightarrow \infty} \frac{1}{t} \log \mathbf{E} \mathrm{e}^{\lambda\left|C_{1}(t)\right|} \leqq \lambda^{2}
$$

Theorem 1(i) follows from (2.6) and (2.10).

Remark. Computations very similar to those of $[1]$, but related to the range of a one dimensional Brownian bridge rather then the range of the Wiener process have been carried out [2].

\section{Proof of the lower Bounds in Theorem 1 (ii) and 1 (iii)}

By Jensen's inequality

$$
\mathbf{E} \mathrm{e}^{\lambda\left|\boldsymbol{C}_{1}(t)\right|} \geqq \mathrm{e}^{\lambda \mathbf{E}\left|\mathcal{C}_{1}(t)\right|}
$$

Hence

$$
\frac{1}{t} \log \mathbf{E e}^{\lambda\left|C_{1}(t)\right|} \geqq \lambda \frac{\mathbf{E}\left|C_{1}(t)\right|}{t} .
$$


For $d=3,4, \ldots$ we have (see [1], [6], [8])

$$
\lim _{t \rightarrow \infty} \frac{\mathbf{E}\left|C_{1}(t)\right|}{t}=C(d),
$$

where $C(d)$ is the Newtonian capacity of the unit ball in $\mathbf{R}^{d}$ :

$$
C(d)=d(d-2) \omega_{d}
$$

Combining (3.1)-(3.4) we obtain

$$
S(\lambda) \geqq \lambda d(d-2) \omega_{d}, \quad d=3,4, \ldots
$$

Furthermore, by (1.11)

$$
\mathbf{E e}^{\lambda\left|C_{1}(t)\right|} \geqq \mathrm{e}^{\lambda \omega_{d}} \mathbf{E e}^{\lambda \omega_{d-2} m(t)}
$$

Define

$$
\Phi(\xi, t)=\mathscr{P}\{m(t)>\xi\} .
$$

By Levy's maximal inequality (Theorem 3.6.5 of [7])

$$
\begin{aligned}
\Phi(\xi, t) \leqq 2 \mathscr{P}\{|\beta(t)|>\xi\} & =\frac{2}{(4 \pi t)^{d / 2}} \int_{|x|>\xi} \mathrm{e}^{-|x|^{2} /(4 t)} \mathrm{d} x \\
& =\frac{2}{\Gamma(d / 2)} \int_{\xi^{2} /(4 t)}^{\infty} \mathrm{e}^{-q} q^{(d-2) / 2} \mathrm{~d} q .
\end{aligned}
$$

Define for $\xi>0$ and $x \in \mathbf{R}^{d}$

$$
B_{\xi}(x)=\left\{y \in \mathbf{R}^{d}|| x-y \mid<\xi\right\}
$$

and

$$
H(\xi)=\left\{x \in \mathbf{R}^{d} \mid x_{1}<\xi\right\}
$$

Then

$$
\begin{aligned}
\Phi(\xi, t) & =\mathscr{P}\left\{\exists s \in[0, t]: \beta(s) \in B_{\xi}^{c}(0)\right\} \\
& \geqq \mathscr{P}\left\{\exists s \in[0, t]: \beta(s) \in H^{c}(\xi)\right\}=\frac{1}{(\pi t)^{1 / 2}} \int_{\xi}^{\infty} \mathrm{e}^{-q^{2} /(4 t)} \mathrm{d} q .
\end{aligned}
$$

But

$$
\mathbf{E e}^{\lambda \omega_{d-1} m(t)}=-\int_{0}^{\infty} \mathrm{d} \xi \mathrm{e}^{\lambda \omega_{d-1} \xi} \frac{\partial \Phi}{\partial \xi}(\xi, t),
$$

so that integration by parts together with (3.8) yield

$$
\mathbf{E} \mathrm{e}^{\lambda \omega_{d-1} m(t)}=1+\lambda \omega_{d-1} \int_{0}^{\infty} \mathrm{d} \xi \mathrm{e}^{\lambda \omega_{d-1} \xi} \Phi(\xi, t) .
$$


By (3.11)

$$
\begin{aligned}
\mathrm{Ee}^{\lambda \omega_{d-1} m(t)} \geqq 1+\int_{0}^{\infty} \mathrm{d} \xi \lambda \omega_{d-1}(\pi t)^{-1 / 2} \mathrm{e}^{\lambda \omega_{d-1} \xi} \int_{\xi}^{\infty} \mathrm{d} q \mathrm{e}^{-q^{2} /(4 t)} \\
=(\pi t)^{-1 / 2} \int_{0}^{\infty} \mathrm{d} \xi \mathrm{e}^{\lambda \omega_{d-1} \xi-\xi^{2} /(4 t)} \geqq(\pi t)^{-1 / 2} \int_{2 t \lambda \omega_{d-1}}^{\infty} \mathrm{d} \xi \\
\cdot \mathrm{e}^{\lambda \omega_{d-1} \xi^{\xi} \xi^{2} /(4 t)}=\mathrm{e}^{\lambda^{2} \omega_{d-1}^{2} t}
\end{aligned}
$$

Hence

$$
S(\lambda)=\liminf _{t \rightarrow \infty} \frac{1}{t} \log \mathbf{E}^{\lambda\left|C_{1}(t)\right|} \geqq \lambda^{2} \omega_{d-1}^{2},
$$

and the lower bounds in Theorem 1 (ii) and 1 (iii) follow from (3.5) and (3.15).

\section{The upper bounds}

\subsection{The basic construction}

Let us fix a positive number, $y>0$, and define the following sequence of consecutive stopping times of the Wiener process $s \mapsto w(s)$ :

$$
\begin{aligned}
\theta_{0}^{y} & =0, \\
\theta_{n+1}^{y} & =\inf \left\{s>\theta_{n}^{y}|| w(s)-w\left(\theta_{n}^{y}\right) \mid>y\right\} .
\end{aligned}
$$

I.e.: at the time $\theta_{n}^{y}$ we fix a ball of radius $y$ centred at the actual position, $w\left(\theta_{n}^{y}\right)$, of the Wiener process, and $\theta_{n+1}^{y}$ is defined as the first hitting time of this ball. Clearly, the consecutive time intervals

$$
\tau_{n}^{y}=\theta_{n}^{y}-\theta_{n-1}^{y}, \quad n \geqq 1
$$

are independent identically distributed and their common distribution is that of the hitting time of a ball of radius $y$ by the Wiener process.

We denote by $v^{y}$ the renewal process

$$
v^{y}(t)=\max \left\{n \in \mathbf{N} \mid \theta_{n}^{y} \leqq t\right\}
$$

The geometric construction we are going to use is the following: we place a ball of radius $y+1$ centred at each one of the consecutive positions of the Wiener process at the stopping times $\theta_{n}^{y}, n=0,1,2, \ldots, v^{y}(t)$. The following setinclusion is evident

$$
C(t) \subset \bigcup_{k=0}^{v^{y}(t)} B_{1+y}\left(w\left(\theta_{k}^{y}\right)\right)
$$

Hence we get an upper bound to the volume of the Wiener sausage:

$$
|C(t)| \leqq \omega_{d}(1+y)^{d}+h(y) v^{y}(t),
$$


where $h(y)$ is half of the volume of the symmetric difference of two balls of radii $y+1$ with the centres displaced by a distance $y$. By elementary geometry we get the expression

$$
h(y)=2 \omega_{d-1}(1+y)^{d} \int_{0}^{\frac{y}{2(1+y)}}\left(1-z^{2}\right)^{\frac{d-1}{2}} \mathrm{~d} z .
$$

Since the left hand side of (4.5) is independent of $y$, we conclude that

$$
S(\lambda) \leqq \inf _{y>0} \lim _{t \rightarrow \infty} \frac{1}{t} \log \mathbf{E}^{\lambda h(y) v y(t)} .
$$

The existence of the limit on the right hand side easily follows again from a subadditivity argument (see the proof of Lemma 4.1(ii)).

We want to exploit once more the scaling properties of the Wiener process: the processes

$$
t \mapsto v^{y}(t)
$$

and

$$
t \mapsto v^{1}\left(t / y^{2}\right) \equiv v\left(t / y^{2}\right)
$$

have the same distribution, and consequently (4.7) is equivalent to

$$
S(\lambda) \leqq \inf _{y>0} \frac{1}{y^{2}} \psi(\lambda h(y))
$$

with $\psi: \mathbf{R} \rightarrow \mathbf{R}$ defined by

$$
\psi(\lambda)=\lim _{t \rightarrow \infty} \frac{1}{t} \log \mathbf{E}^{\lambda v(t)} .
$$

\subsection{A large deviation principle for renewal processes}

We are going to find an expression for $\psi(\lambda)$ defined in (4.9). Let $\tau_{k}, k=1,2, \ldots$ be independent identically distributed non-negative random variables and define the renewal process $t \mapsto v(t)$ in the usual way:

$$
v(t)=\max \left\{n \in \mathbf{N} \mid \sum_{k=1}^{n} \tau_{k} \leqq t\right\} .
$$

Define the logarithmic cumulant generating function

$$
\phi(\lambda)=\log \mathbf{E e}^{\lambda \tau_{1}} .
$$


$\phi(\lambda)$ is finite and real-analytic at least for $\lambda \in(-\infty, 0)$. By virtue of Cramér's Theorem the large deviations of the sums $T_{n}=\sum_{k=1}^{n} \tau_{k}$ of the independent identically distributed random variables are governed by the rate function

$$
I(x)=\sup _{\lambda \in \mathbf{R}}(\lambda x-\phi(\lambda)) \in \mathbf{R}_{+} \cup\{\infty\} .
$$

(Note that in this case $I(x)=\infty$ for $x<0$ and the level sets of $I$ are not necessarily compact: if $\phi(\lambda)=\infty$ for $\lambda>0$ then $\lim _{x \rightarrow \infty} I(x)=0$.)

Lemma 4.1 states a large deviation principle for the renewal process $v(t)$.

Lemma 4.1. (i) For any subset $A \in \mathbf{R}$ the following asymptotic bounds hold

$$
\begin{aligned}
-\inf _{x \in \mathscr{A}} J(x) & \leqq \liminf _{t \rightarrow \infty} \frac{1}{t} \log \mathscr{P}\left(\frac{\nu(t)}{t} \in \mathscr{A}\right) \\
& \leqq \limsup _{t \rightarrow \infty} \frac{1}{t} \log \mathscr{P}\left(\frac{v(t)}{t} \in \bar{A}\right) \leqq-\inf _{x \in \bar{A}} J(x),
\end{aligned}
$$

with the rate function $J$ given by

$$
J(x)=x I(1 / x) .
$$

(ii) The limit

$$
\psi(\lambda)=\lim _{t \rightarrow \infty} \frac{1}{t} \log \mathbf{E} \mathrm{e}^{\lambda v(t)}
$$

exists and is given by

$$
\psi(\lambda)=-\phi^{-1}(-\lambda) .
$$

Remark. Lemma 4.1 is probably well known, but as we did not find it explicitly stated anywhere, we were obliged to state it ourselves.

Proof. (i) By definition of process $v(t)$ we have the following identities:

$$
\begin{aligned}
& \{v(t)<y\}=\left\{T_{\lceil y]}>t\right\}, \\
& \{v(t) \leqq y\}=\left\{T_{\lfloor y\rfloor+1}>t\right\}, \\
& \{v(t)>y\}=\left\{T_{\lfloor y\rfloor+1} \leqq t\right\}, \\
& \{v(t) \geqq y\}=\left\{T_{\lceil y\rceil} \leqq t\right\},
\end{aligned}
$$

where

$$
\begin{aligned}
& \lceil y\rceil=\min \{n \in \mathbf{Z} \mid n \geqq y\}, \\
& \lfloor y\rfloor=\max \{n \in \mathbf{Z} \mid n \leqq y\} .
\end{aligned}
$$


We prove the asymptotic bound (4.13) for semi-infinite intervals of the form $(-\infty, x),(-\infty, x],(x, \infty),[x, \infty)$, where $x \in(0, \infty)$. For example:

$$
\begin{aligned}
\liminf _{t \rightarrow \infty} \frac{1}{t} \log \mathscr{P}\left(\frac{v(t)}{t}<x\right) & =\liminf _{t \rightarrow \infty} \frac{1}{t} \log \mathscr{P}\left(T_{\lceil t x\rceil}>t\right) \\
& =\liminf _{t \rightarrow \infty} \frac{\lceil t x\rceil}{t} \frac{1}{\lceil t x\rceil} \log \mathscr{P}\left(\frac{\left.T_{\lceil t x\rceil}>\frac{t}{\lceil t x\rceil}\right)}{\lceil t x\rceil}\right) \\
& \geqq x \liminf _{m \rightarrow \infty} \frac{1}{m} \log \mathscr{P}\left(\frac{T_{m}}{m}>\frac{1}{x}+\varepsilon\right) \geqq-x \inf _{y>\frac{1}{x}+\varepsilon} I(y),
\end{aligned}
$$

for any $\varepsilon>0$. Cramér's Theorem has been used in the last inequality. Using convexity and continuity of $I$ in the interval $(0, \infty)$ we finally get:

$$
\liminf _{t \rightarrow \infty} \frac{1}{t} \log \mathscr{P}\left(\frac{v(t)}{t}<x\right) \geqq-\inf _{y<x} y I\left(\frac{1}{y}\right) .
$$

In exactly the same fashion we get

$$
\begin{aligned}
& \liminf _{t \rightarrow \infty} \frac{1}{t} \log \mathscr{P}\left(\frac{v(t)}{t}>x\right) \geqq-\inf _{y>x} y I\left(\frac{1}{y}\right), \\
& \liminf _{t \rightarrow \infty} \frac{1}{t} \log \mathscr{P}\left(\frac{v(t)}{t} \leqq x\right) \geqq-\inf _{y \geqq x} y I\left(\frac{1}{y}\right), \\
& \liminf _{t \rightarrow \infty} \frac{1}{t} \log \mathscr{P}\left(\frac{v(t)}{t} \geqq x\right) \geqq-\inf _{y \geqq x} y I\left(\frac{1}{y}\right) .
\end{aligned}
$$

Passing from the semi-infinite intervals to general subsets $A \subset \mathbf{R}$ is done exactly in the same way as in the proof of Cramér's Theorem (see e.g. [11]).

(ii) The existence of the limit (4.15) follows from a subadditivity argument. In (4.20) below we shall use the auxiliary notation:

and

$$
v\left(t_{1}, t_{2}\right)=\#\left\{k \mid t_{1}<T_{k} \leqq t_{2}\right\},
$$

$$
q(t)=\mathbf{E}^{\lambda v(t)}
$$

With this notation

$$
\begin{aligned}
q(t+s) & =\mathbf{E} \mathrm{e}^{\lambda v(t+s)}=\mathbf{E}\left(\mathrm{e}^{\lambda(v(t)+1)} \mathrm{e}^{\lambda v\left(T_{v(t)+1}, t+s\right)}\right) \\
& =\mathrm{e}^{\lambda} \mathbf{E}\left(\mathrm{e}^{\lambda v(t)} q\left(t+s-T_{v(t)+1}\right)\right) \leqq \mathrm{e}^{\lambda} \mathbf{E}\left(\mathrm{e}^{\lambda v(t)} q(s)\right) \\
& =\mathrm{e}^{\lambda} q(t) q(s) .
\end{aligned}
$$

In the second equality the strong Markov property of the discrete time process $T_{n}, n \geqq 0$ has been used. In the last inequality we exploited the obvious monotonicity in $t$ of $\mathbf{E} \exp \lambda \nu(t)$. 
The existence of

$$
\psi(\lambda)=\lim _{t \rightarrow \infty} \frac{1}{t} \log \mathbf{E} \mathrm{e}^{\lambda v(t)}=\inf _{t>0} \frac{1}{t} \log \mathbf{E} \mathrm{e}^{\lambda v(t)}
$$

follows from (4.20) via a standard subadditivity argument. Given the existence of the limit (4.15) and the large deviation principle (4.13)-(4.14) we can apply the Laplace-Varadhan asymptotic formula

$$
\psi(\lambda)=\sup _{x \in \mathbf{R}}(\lambda x-J(x))=\sup _{x \in \mathbf{R}}(\lambda x-x I(1 / x)) .
$$

Since the Legendre transform is an involution in the class of convex and lower semicontinuous functions, it is sufficient in order to get (4.16) that

$$
\sup _{\lambda \in \mathbf{R}}\left(\lambda x+\phi^{-1}(-\lambda)\right)=x I(1 / x) \text {. }
$$

This is straightforward:

$$
\begin{aligned}
\sup _{\lambda \in \mathbf{R}}\left(\lambda x+\phi^{-1}(-\lambda)\right) & =\sup _{\lambda \in \mathbf{R}}\left(-\lambda x+\phi^{-1}(\lambda)\right)=\sup _{\lambda^{\prime} \in \mathbf{R}}\left(\lambda^{\prime}-x \phi\left(\lambda^{\prime}\right)\right) \\
& =x \sup _{\lambda^{\prime} \in \mathbf{R}}\left(\lambda^{\prime} / x-\phi\left(\lambda^{\prime}\right)\right)=x I(1 / x) .
\end{aligned}
$$

In the second equality we have used the fact that $\phi$ is monotonic increasing and, consequently, invertible.

This proves Lemma 4.1 .

\subsection{Proof of the upper bounds in Theorem 1(ii) and 1(iii)}

By (4.8) and (4.16)

$$
S(\lambda) \leqq \inf _{y>0}\left\{-\frac{1}{y^{2}} \phi^{-1}(-\lambda h(y))\right\}
$$

where $\phi$ is defined in (4.11), $\tau$ being the hitting time of the unit sphere of the Wiener process.

By Theorem 2 of [12] we have

$$
\begin{aligned}
-\phi(-p) & =\log \left\{\frac{2^{(d-2) / 2} \Gamma(d / 2)}{p^{(d-2) / 4}} I_{(d-2) / 2}\left(p^{1 / 2}\right)\right\} \\
& =\log \left\{\frac{\Gamma(d / 2)}{\pi^{1 / 2} \Gamma((d-1) / 2)} \int_{0}^{\pi} \mathrm{e}^{p^{1 / 2} \cos \theta}(\sin \theta)^{d-2} \mathrm{~d} \theta\right\} .
\end{aligned}
$$

Lemma 4.2. For $p \geqq 0$ and $d=2,3, \ldots$

$$
-\phi(-p) \geqq \log \left(1+\frac{p}{2 d}\right) .
$$


Proof. For $p \geqq 0$ and $d=2,3, \ldots$

$$
\begin{aligned}
& \int_{0}^{\pi} \mathrm{e}^{p^{1 / 2} \cos \theta}(\sin \theta)^{d-2} \mathrm{~d} \theta=2 \int_{0}^{\pi / 2} \cosh \left(p^{1 / 2} \cos \theta\right)(\sin \theta)^{d-2} \mathrm{~d} \theta \\
\geqq & \int_{0}^{\pi / 2}\left(1+\frac{p(\cos \theta)^{2}}{2}\right)(\sin \theta)^{d-2} \mathrm{~d} \theta=\frac{\pi^{1 / 2} \Gamma((\mathrm{d}-1) / 2)}{\Gamma(\mathrm{d} / 2)}\left(1+\frac{p}{2 \mathrm{~d}}\right) .
\end{aligned}
$$

Substituting (4.28) into (4.26) gives (4.27).

To complete the proof of Theorem 1 we invert (4.27):

$$
-\phi^{-1}(-p) \leqq 2 d\left(\mathrm{e}^{p}-1\right), \quad p \geqq 0 .
$$

Hence by (4.25)

$$
S(\lambda) \leqq 2 d \inf _{y>0} \frac{1}{y^{2}}\left(\mathrm{e}^{\lambda h(y)}-1\right) \leqq 2 d \inf _{y>0} \frac{1}{y^{2}}\left(\mathrm{e}^{\lambda g(y)}-1\right),
$$

where $g:[0, \infty) \rightarrow[0, \infty)$ is defined by

$$
g(y)=\omega_{d-1} y(1+y)^{d-1} .
$$

For $0 \leqq \lambda \leqq 1$ we have by $(4.30)$

$$
S(\lambda) \leqq 2 d\left(\mathrm{e}^{\lambda g(1)}-1\right) \leqq 2 d g(1) \mathrm{e}^{g(1)} \lambda .
$$

For $\lambda \geqq 1$ we have by $(4.30)$

$$
S(\lambda) \leqq 2 d \lambda^{2}\left(\mathrm{e}^{\lambda g(1 / \lambda)}-1\right) \leqq 2 d\left(\mathrm{e}^{g(1)}-1\right) \lambda^{2} \leqq 2 d g(1) \mathrm{e}^{g(1)} \lambda^{2} .
$$

Combining (4.32) and (4.33) gives

$$
S(\lambda) \leqq 2 d g(1) \mathrm{e}^{g(1)} \max \left\{\lambda, \lambda^{2}\right\} \leqq d 2^{d} \omega_{d-1} \mathrm{e}^{2^{d-1} \omega_{d-1}} \max \left\{\lambda, \lambda^{2}\right\} .
$$

Acknowledgement. The work of B.T. was supported by the British Science and Engineering Research Council.

\section{References}

1. Berezhkovskii, A.M., Makhnovskii, Yu.A., Suris, R.A.: Wiener sausage volume moments. J. Stat. Phys. 57, 333-346 (1989)

2. Berg, M. van den, Lewis, J.T.: On the asymptotics of a Wiener integral. Proc. R. Soc. Edinb. 105 A, 195-198 (1987)

3. Ciesielski, Z., Taylor, S.J.: First passage time and sojourn density for Brownian motion in space and the exact Hausdorff measure of the sample path. Trans. Am. Math. Soc. 103, 434-450 (1962)

4. Chan, D.Y.C., Hughes, B.D.: On a random process interpolating between Markovian and non-Markovian random walks. J. Phys. A: Math. Gen. 17, L121-L127 (1984)

5. Donsker, M.D., Varadhan, S.R.S.: Asymptotics of the Wiener sausage. Commun. Pure Appl. Math. 28, 525-565(1975)

6. Le Gall, J.F.: Sur une conjecture de M. Kac. Probab. Th. Rel. Fields 78, 389-402 (1988)

7. Simon, B.: Functional integration and quantum physics. New York: Academic Press 1979

8. Spitzer, F.: Electrostatic capacity, heat flow and Brownian motion. Z. Wahrscheinlichkeitstheor. Verw. Geb. 3, 110-121 (1964)

9. Sznitman, A.S.: Some bounds and limiting results for the measure of Wiener sausage of small radius associated with elliptic diffusions. Stochastic Processes Appl. 25, 1-25 (1987)

10. Sznitman, A.S.: Lifshitz tail and Wiener sausage I., II. J. Funct. Anal. 94, 223-246; 247-272 (1990)

11. Varadhan, S.R.S.: Large Deviations and Applications. CBMS-NSF, Regional Conference Series in Applied Mathematics 46. Philadelphia: SIAM 1984

12. Wendel, J.G.: Hitting spheres with Brownian motion. Ann. Probab. 8, 164-169 (1980) 